doi:10.13108/2020-12-2-56

\title{
FUNDAMENTAL OPERATOR FUNCTIONS OF INTEGRO-DIFFERENTIAL OPERATORS UNDER SPECTRAL OR POLYNOMIAL BOUNDEDNESS
}

\author{
M.V. FALALEEV
}

\begin{abstract}
We study a Cauchy problem for a degenerate higher order integro-differential equation in Banach spaces. The operator kernel of the integral part of the equation is a linear combination of the operator coefficients of its differential part, which corresponds to the physical meaning of some technological processes. The solution is constructed in the space of generalized functions (distributions) in Banach spaces using the methods of the theory of fundamental operands. The convolutional representation of the original equation leads to a further active use of the convolutional technique and its properties. For the considered equations, the corresponding fundamental operator functions are constructed. By means of this operator, a unique generalized solution to the original Cauchy problem in the class of distributions with a left-bounded support is recovered. The analysis of the resulting generalized solution allows us to study the solvability problem in the classical sense. The fundamental operator function is constructed in terms of the theory of semigroups of operators with kernels. Abstract results are illustrated by examples of initial-boundary value problems from visco-elasticity theory.
\end{abstract}

Keywords: Banach space, generalized function, distribution, fundamental operatorfunction, integro-differential operator, spectral boundedness, polynomial boundedness

Mathematics Subject Classification:: 34G10, 45K05, 45N05

\section{INTRODUCTION}

For correct modeling of some natural or technological processes one needs to take into consideration not only a momentary influence of determining factors, but also an observation history. Such phenomena arise, for instance, in studying the oscillations of membranes in oil media, when the perturbation from the membrane spreads to the media and then goes back in some changed form due to dynamical processes generated by the membrane itself. One of the ways to describe such models is the theory of integro-partial differential equations of convolution type. Among latter equations, a special place belongs to the equations with a non-invertible operator at a higher in time derivative, since the initial boundary value problems for such equations are solvable in the classes of functions of finite smoothness not for all combinations of initial and boundary conditions and the free term (right hand side) in the equation. The solutions in the class of distributions have no such disadvantages and the problem can be formulated in the most general form and to be solved via a reduction to a degenerate integro-differential equations in Banach spaces. The most effective approach for constructing generalized solutions of degenerate integro-differential equations is the theory of fundamental operator functions, see [8], according to which the sought generalized solution is recovered as a convolution of a fundamental operator function and a generalized function involving all data of the problem. Analyzing the generalized solutions constructed in this way, one can make conclusions on

M.V. FAlaleEv, Fundamental operator-FunCtions of INTEGro-DifFERENTIAL OPERATORS UNDER SPECTRAL OR POLYNOMIAL BOUNDEDNESS.

(C) Falaleev M.V. . 2020.

Submitted September 20, 2019. 
the existence and uniqueness of the classical smooth solution, its structure, properties and a possibility of numerical experiments.

In the present work we consider integro-differential equations, in which the operator kernel of the integral part is a linear combination of operator coefficients in its differential part. Physically this means that at all times, the same factors influence the system state.

We study the following Cauchy problems:

$$
\begin{aligned}
& B u^{(N)}(t)-A u(t)-\int_{0}^{t}(\alpha(t-s) A+\beta(t-s) B) u(s) d s=f(t), \\
& u(0)=u_{0}, \quad u^{\prime}(0)=u_{1}, \quad \ldots, \quad u^{(N-1)}(0)=u_{N-1},
\end{aligned}
$$

where $B \in \mathcal{L}\left(E_{1}, E_{2}\right)$ is non-invertible, $A$ is a closed linear operator from $E_{1}$ into $E_{2}$, and $E_{1}$ and $E_{2}$ are Banach spaces. The operator $A$ is spectrally relatively bounded with respect to $B$, see [4, 9];

$$
\begin{aligned}
& B u^{(2 N)}(t)-A_{1} u^{(N)}(t)-A_{0} u(t) \\
& \quad-\int_{0}^{t}\left(\alpha_{1}(t-s) A_{1}+\alpha_{0}(t-s) A_{0}+\beta(t-s) B\right) u(s) d s=f(t), \\
& u(0)=u_{0}, \quad u^{\prime}(0)=u_{1}, \quad \ldots \quad u^{(2 N-1)}(0)=u_{2 N-1} .
\end{aligned}
$$

Here $B, A_{1}, A_{0} \in \mathcal{L}\left(E_{1}, E_{2}\right), B$ is non-invertible, the pair of the operators $\left(A_{1}, A_{0}\right)$ is polynomially relatively bounded with respect to the operator $B$, see [3].

If in equations (1) and (3) the scalar functions $\alpha(t), \beta(t), \alpha_{0}(t), \alpha_{1}(t)$ are identically zero, the solvability theory of Cauchy problems (1)-(2) and (3)-(4) under the mentioned conditions in the classes of functions of finite smoothness was well-developed in [3], 4, , 9].

In the present work we construct solutions in the class $K_{+}^{\prime}\left(E_{1}\right)$ of distributions with leftbounded supports. This class is natural due to a series of reasons. First, a solution to the Cauchy problem is defined on the ray $t \geqslant 0$, second, while constructing the solutions, we use the convolution of generalized functions, which is always well-defined in this class, and third, the convolution is associative in this class, which is very needed for all made transformations.

\section{FUNDAMENTAL OPERATOR FUNCTIONS UNDER SPECTRAL BOUNDEDNESS}

Let $E_{1}, E_{2}$ be Banach spaces, an operator $B \in L\left(E_{1}, E_{2}\right)$ be non-invertible, $A$ be a closed linear operator acting from $E_{1}$ into $E_{2}$. Following works [4], [9], as a $B$-resolvent set of the operator $A$ we call the following open set in the complex plane

$$
\rho^{B}(A) \equiv\left\{\mu \in C:(\mu B-A)^{-1} \in L\left(E_{2}, E_{1}\right)\right\} .
$$

The operator $A$ is called spectrally relatively bounded with respect to the operator $B$ (or $(B, \sigma)$-bounded) if outside some circle of a radius $a>0$ the operator pencil $(\mu B-A)$ is continuously invertible, that is, $\{\mu \in C:|\mu|>a\} \subset \rho^{B}(A)$. We consider a circumference $\Gamma \equiv\{\mu \in C:|\mu|=r>a\}$ in the complex plane, then, as it was shown in works [4], 9], under a $(B, \sigma)$-boundedness, the operators

$$
P=\frac{1}{2 \pi i} \oint_{\Gamma}(\mu B-A)^{-1} B d \mu, \quad Q=\frac{1}{2 \pi i} \oint_{\Gamma} B(\mu B-A)^{-1} d \mu
$$

are projectors in $E_{1}$ and $E_{2}$, respectively. The projectors $P$ and $Q$ give rise to the expansions of the space into direct sums $E_{1} \equiv E_{1}^{0} \oplus E_{1}^{1}=\operatorname{Ker} P \oplus \operatorname{Im} P$ and $E_{2} \equiv E_{2}^{0} \oplus E_{2}^{1}=\operatorname{Ker} Q \oplus \operatorname{Im} Q$. The actions of the operators $A$ and $B$ are naturally split so that they restrictions $A_{0}: E_{1}^{0} \rightarrow E_{2}^{0}$ 
and $B_{1}: E_{1}^{1} \rightarrow E_{2}^{1}$ are continuously invertible, $A_{1}: E_{1}^{1} \rightarrow E_{2}^{1}$ is bounded, the operators $A$ and $B$ pseudocommute with the projectors $P$ and $Q$, that is, $Q B=B P$ and $Q A=A P$.

In what follows we denote: $\alpha(t), \beta(t) \in C(t \geqslant 0), \Lambda(t)$ is the resolvent of the kernel $(-\alpha(t) \theta(t)), f_{n}(t)=\frac{t^{n-1}}{(n-1) !} \theta(t), n \in \mathbb{N}, \theta(t)$ is the Heaviside function [1], 2], $R(t)$ is the resolvent of the convolution kernel $k(t) \theta(t)=f_{N}(t) * \beta(t) \theta(t), N$ is a natural number, $f_{0}(t)=\delta(t)$, and $\delta(t)$ is the Dirac delta-function [1], 2].

Theorem 1. If the operator $A$ is spectrally relatively bounded with respect to $B$, then an integro-differential operator

$$
B \delta^{(N)}(t)-A \delta(t)-(\alpha(t) A+\beta(t) B) \theta(t)=B\left(\delta^{(N)}(t)-\beta(t) \theta(t)\right)-A(\delta(t)+\alpha(t) \theta(t))
$$

possesses a fundamental operator function

$$
\mathcal{E}_{N}(t)=B_{1}^{-1} \mathcal{U}_{N}(t) Q-\mathcal{V}_{N}(t)(I-Q)
$$

in the class $K_{+}^{\prime}\left(E_{2}\right)$ of generalized functions with left-bounded supports. Here

$$
\begin{aligned}
& \mathcal{U}_{N}(t)=\sum_{k=1}^{\infty} f_{N \cdot k}(t) *(\delta(t)+R(t) \theta(t))^{k} *(\delta(t)+\alpha(t) \theta(t))^{k-1}\left(A_{1} B_{1}^{-1}\right)^{k-1} \\
& \mathcal{V}_{N}(t)=\sum_{q=0}^{\infty}\left(A_{0}^{-1} B_{0}\right)^{q} A_{0}^{-1}\left(\delta^{(N)}(t)-\beta(t) \theta(t)\right)^{q} *(\delta(t)+\Lambda(t) \theta(t))^{q+1}
\end{aligned}
$$

Hereinafter by the $k$ th power of a generalized function we mean its $k$-multiple convolution with itself, the zero power of a generalized function is $\delta(t)$.

Proof. According the definition of a fundamental operator function [8], we are going to check the validity of two convolution identities:

$$
\begin{aligned}
& {\left[B\left(\delta^{(N)}(t)-\beta(t) \theta(t)\right)-A(\delta(t)+\alpha(t) \theta(t))\right] * \mathcal{E}_{N}(t)=I_{2} \delta(t) \quad \text { on } \quad K_{+}^{\prime}\left(E_{2}\right),} \\
& \mathcal{E}_{N}(t) *\left[B\left(\delta^{(N)}(t)-\beta(t) \theta(t)\right)-A(\delta(t)+\alpha(t) \theta(t))\right]=I_{1} \delta(t) \quad \text { on } \quad K_{+}^{\prime}\left(E_{1}\right) .
\end{aligned}
$$

It is known that the family of functions $f_{n}(t)$ satisfies the convolution identities [1], [2]

$$
f_{n}(t) * f_{k}(t)=f_{n+k}\left(t, \quad \delta^{(n)}(t) * f_{n}(t)=\delta(t) .\right.
$$

Hence,

$$
\begin{aligned}
B\left(\delta^{(N)}(t)\right. & -\beta(t) \theta(t)) * B_{1}^{-1} \mathcal{U}_{N}(t) Q \\
= & B\left(\delta^{(N)}(t)-\beta(t) \theta(t)\right) * B_{1}^{-1} f_{N}(t) *(\delta(t)+R(t) \theta(t)) \\
& * \sum_{k=1}^{\infty} f_{N \cdot(k-1)}(t) *(\delta(t)+R(t) \theta(t))^{k-1} *(\delta(t)+\alpha(t) \theta(t))^{k-1}\left(A_{1} B_{1}^{-1}\right)^{k-1} Q \\
= & B B_{1}^{-1}(\delta(t)-k(t) \theta(t)) *(\delta(t)+R(t) \theta(t)) \\
& * \sum_{k=1}^{\infty} f_{N \cdot(k-1)}(t) *(\delta(t)+R(t) \theta(t))^{k-1} *(\delta(t)+\alpha(t) \theta(t))^{k-1}\left(A_{1} B_{1}^{-1}\right)^{k-1} Q \\
= & Q \delta(t)+\sum_{k=1}^{\infty} f_{N \cdot k}(t) *(\delta(t)+R(t) \theta(t))^{k} *(\delta(t)+\alpha(t) \theta(t))^{k}\left(A_{1} B_{1}^{-1}\right)^{k} Q,
\end{aligned}
$$


and

$$
\begin{aligned}
A(\delta(t) & +\alpha(t) \theta(t)) * B_{1}^{-1} \mathcal{U}_{N}(t) Q \\
& =\sum_{k=1}^{\infty} f_{N \cdot k}(t) *(\delta(t)+R(t) \theta(t))^{k} *(\delta(t)+\alpha(t) \theta(t))^{k}\left(A_{1} B_{1}^{-1}\right)^{k} Q
\end{aligned}
$$

that is,

$$
\left[B\left(\delta^{(N)}(t)-\beta(t) \theta(t)\right)-A(\delta(t)+\alpha(t) \theta(t))\right] * B_{1}^{-1} \mathcal{U}_{N}(t) Q=Q \delta(t) .
$$

In the same way we find:

$$
\begin{aligned}
& B\left(\delta^{(N)}(t)-\beta(t) \theta(t)\right) * \mathcal{V}_{N}(t)(I-Q) \\
& =\sum_{q=0}^{\infty} B_{0}\left(A_{0}^{-1} B_{0}\right)^{q} A_{0}^{-1}\left(\delta^{(N)}(t)-\beta(t) \theta(t)\right)^{q+1} *(\delta(t)+\Lambda(t) \theta(t))^{q+1}(I-Q),
\end{aligned}
$$

and

$$
\begin{aligned}
& A(\delta(t)+\alpha(t) \theta(t)) * \mathcal{V}_{N}(t)(I-Q) \\
& =\sum_{q=0}^{\infty} A_{0}\left(A_{0}^{-1} B_{0}\right)^{q} A_{0}^{-1}\left(\delta^{(N)}(t)-\beta(t) \theta(t)\right)^{q} *(\delta(t)+\Lambda(t) \theta(t))^{q}(I-Q) \\
& =(I-Q) \delta(t)+\sum_{q=1}^{\infty} B_{0}\left(A_{0}^{-1} B_{0}\right)^{q-1} A_{0}^{-1}\left(\delta^{(N)}(t)-\beta(t) \theta(t)\right)^{q} *(\delta(t)+\Lambda(t) \theta(t))^{q}(I-Q) \\
& =(I-Q) \delta(t)+\sum_{q=0}^{\infty} B_{0}\left(A_{0}^{-1} B_{0}\right)^{q} A_{0}^{-1}\left(\delta^{(N)}(t)-\beta(t) \theta(t)\right)^{q+1} *(\delta(t)+\Lambda(t) \theta(t))^{q+1}(I-Q)
\end{aligned}
$$

Therefore,

$$
\left[B\left(\delta^{(N)}(t)-\beta(t) \theta(t)\right)-A(\delta(t)+\alpha(t) \theta(t))\right] * \mathcal{V}_{N}(t)(I-Q)=-(I-Q) \delta(t),
$$

and hence,

$$
\left[B\left(\delta^{(N)}(t)-\beta(t) \theta(t)\right)-A(\delta(t)+\alpha(t) \theta(t))\right] * \mathcal{E}_{N}(t)=Q \delta(t)+(I-Q) \delta(t)=I \delta(t) .
$$

This completes the proof of the first identity.

We proceed to proving the second identity. First we find that

$$
\begin{array}{rl}
B_{1}^{-1} \mathcal{U}_{N}(t) Q & * B\left(\delta^{(N)}(t)-\beta(t) \theta(t)\right) \\
= & B_{1}^{-1} \sum_{k=1}^{\infty} f_{N \cdot(k-1)}(t) *(\delta(t)+R(t) \theta(t))^{k} *(\delta(t)+\alpha(t) \theta(t))^{k-1}\left(A_{1} B_{1}^{-1}\right)^{k-1} B_{1} P \\
& *(\delta(t)-k(t) \theta(t)) \\
= & B_{1}^{-1} \sum_{k=1}^{\infty} f_{N \cdot(k-1)}(t) *(\delta(t)+R(t) \theta(t))^{k-1} *(\delta(t)+\alpha(t) \theta(t))^{k-1}\left(A_{1} B_{1}^{-1}\right)^{k-1} B_{1} P \\
= & P \delta(t)+B_{1}^{-1} \sum_{k=1}^{\infty} f_{N \cdot k}(t) *(\delta(t)+R(t) \theta(t))^{k} *(\delta(t)+\alpha(t) \theta(t))^{k}\left(A_{1} B_{1}^{-1}\right)^{k} B_{1} P
\end{array}
$$




$$
=P \delta(t)+B_{1}^{-1} \sum_{k=1}^{\infty} f_{N \cdot k}(t) *(\delta(t)+R(t) \theta(t))^{k} *(\delta(t)+\alpha(t) \theta(t))^{k}\left(A_{1} B_{1}^{-1}\right)^{k-1} A_{1} P .
$$

Second,

$$
\begin{aligned}
B_{1}^{-1} \mathcal{U}_{N}(t) & Q * A(\delta(t)+\alpha(t) \theta(t)) \\
& =B_{1}^{-1} \sum_{k=1}^{\infty} f_{N \cdot k}(t) *(\delta(t)+R(t) \theta(t))^{k} *(\delta(t)+\alpha(t) \theta(t))^{k}\left(A_{1} B_{1}^{-1}\right)^{k-1} A_{1} P .
\end{aligned}
$$

Hence,

Therefore,

$$
B_{1}^{-1} \mathcal{U}_{N}(t) Q *\left[B\left(\delta^{(N)}(t)-\beta(t) \theta(t)\right)-A(\delta(t)+\alpha(t) \theta(t))\right]=P \delta(t)
$$

$$
\begin{aligned}
\mathcal{V}_{N}(t)(I-Q) & * B\left(\delta^{(N)}(t)-\beta(t) \theta(t)\right) \\
= & \sum_{q=0}^{\infty}\left(A_{0}^{-1} B_{0}\right)^{q+1}\left(\delta^{(N)}(t)-\beta(t) \theta(t)\right)^{q+1} *(\delta(t)+\Lambda(t) \theta(t))^{q+1}(I-P)
\end{aligned}
$$

and

$$
\begin{aligned}
\mathcal{V}_{N}(t)(I-Q) & * A(\delta(t)+\alpha(t) \theta(t)) \\
= & \sum_{q=0}^{\infty}\left(A_{0}^{-1} B_{0}\right)^{q}\left(\delta^{(N)}(t)-\beta(t) \theta(t)\right)^{q} *(\delta(t)+\Lambda(t) \theta(t))^{q}(I-P) \\
= & (I-P) \delta(t) \\
& +\sum_{q=0}^{\infty}\left(A_{0}^{-1} B_{0}\right)^{q+1}\left(\delta^{(N)}(t)-\beta(t) \theta(t)\right)^{q+1} *(\delta(t)+\Lambda(t) \theta(t))^{q+1}(I-P),
\end{aligned}
$$

and this is why

$$
\mathcal{V}_{N}(t)(I-Q) *\left[B\left(\delta^{(N)}(t)-\beta(t) \theta(t)\right)-A(\delta(t)+\alpha(t) \theta(t))\right]=-(I-P) \delta(t) .
$$

This implies the second identity:

$$
\begin{aligned}
\mathcal{E}_{N}(t) & *\left[B\left(\delta^{(N)}(t)-\beta(t) \theta(t)\right)-A(\delta(t)+\alpha(t) \theta(t))\right] \\
& =P \delta(t)+(I-P) \delta(t)=I \delta(t) .
\end{aligned}
$$

Remark 1. If $\alpha(t)=\beta(t) \equiv 0$ in equation (1), then Theorem 1 coincides completely with Theorem 3 in work [5]. As corollaries, this implies corresponding statements in [4], [9], see Theorem 2 and Corollary 2 in work [5].

Remark 2. If $\beta(t) \equiv 0$ in equation (1), then Theorem 1 becomes one of the main statements in work [6], namely, Theorem 1 in this paper.

Remark 3. If $\alpha(t) \equiv 0$ in equation (1), then Theorem 1 becomes the main statement in work [7].

Remark 4. Theorem 1 can be generalized for the case, when the operator $A$ is sectorially or radially relatively bounded with respect to the operator B, see appropriate definitions in [4], 9]. 
Remark 5. In terms of generalized functions, Cauchy problem (1)- (2) is written as follows:

$$
\left[B\left(\delta^{(N)}(t)-\beta(t) \theta(t)\right)-A(\delta(t)+\alpha(t) \theta(t))\right] * \tilde{u}(t)=F(t),
$$

where

$$
F(t)=f(t) \theta(t)+B u_{N-1} \delta(t)+B u_{N-2} \delta^{\prime}(t)+\cdots+B u_{1} \delta^{(N-2)}(t)+B u_{0} \delta^{(N-1)}(t) .
$$

By identity (5), a generalized function $\tilde{u}(t)=\mathcal{E}_{N}(t) * F(t)$ solves equation (7) in the class $K_{+}^{\prime}\left(E_{1}\right)$ and by identity (6) this solution is unique.

If we assume in addition that $\infty$ is a removable singular point of the operator pencil $(\mu B-$ $A)^{-1}$, see [4], [9], that is, $A_{0}^{-1} B_{0} \equiv 0$, then the fundamental operator function $\mathcal{E}_{N}(t)$ casts into the most compact form

$$
\tilde{\mathcal{E}}_{N}(t)=B_{1}^{-1} \mathcal{U}_{N}(t) Q-A_{0}^{-1}(I-Q)(\delta(t)+\Lambda(t) \theta(t)) .
$$

In this case the unique solution of Cauchy problem (1)-(2) in the class $K_{+}^{\prime}\left(E_{1}\right)$ is a regular generalized function $\tilde{u}(t)=\tilde{\mathcal{E}}_{N}(t) * F(t)$ satisfying equation $(1)$. Assuming then that it obeys also initial conditions (2), we obtain the solvability conditions of the Cauchy problem (1)-(2) in the class $C^{N}\left(t \geqslant 0, E_{1}\right)$. By straightforward calculations, for $j=0,1, \ldots, N-1$ we find

$$
\begin{aligned}
\left.\tilde{u}^{(j)}\right|_{t=0}= & -A_{0}^{-1}(I-Q)\left[f^{(j)}(0)+\Lambda(0) f^{(j-1)}(0)+\Lambda^{\prime}(0) f^{(j-2)}(0)+\ldots\right. \\
& \left.+\Lambda^{(j-2)}(0) f^{\prime}(0)+\Lambda^{(j-1)}(0) f(0)\right]+P u_{j} \\
= & u_{j}-\omega_{j},
\end{aligned}
$$

where

$$
\begin{aligned}
w_{j}= & (I-P) u_{j}+A_{0}^{-1}(I-Q)\left[f^{(j)}(0)+\Lambda(0) f^{(j-1)}(0)+\Lambda^{\prime}(0) f^{(j-2)}(0)+\ldots\right. \\
& \left.+\Lambda^{(j-2)}(0) f^{\prime}(0)+\Lambda^{(j-1)}(0) f(0)\right] \\
= & A_{0}^{-1}(I-Q)\left[A u_{j}+f^{(j)}(0)+\Lambda(0) f^{(j-1)}(0)+\Lambda^{\prime}(0) f^{(j-2)}(0)+\ldots\right. \\
& \left.+\Lambda^{(j-2)}(0) f^{\prime}(0)+\Lambda^{(j-1)}(0) f(0)\right] \\
= & A_{0}^{-1}(I-Q) v_{j} .
\end{aligned}
$$

This yields that the function $\tilde{u}(t) \in C^{N}\left(t \geqslant 0, E_{1}\right)$ solves Cauchy problem (1)-(2) if and only if $\omega_{j}=0$ or, by $A_{0}^{-1} \in \mathcal{L}\left(E_{2}, E_{1}\right)$, if $(I-Q) v_{j}=0$.

Thus, we have proved the following theorem.

Theorem 2. If the operator $A$ is spectrally relatively bounded with respect to $B$ and $\infty$ is a removable singular point, then Cauchy problem (1)-(2) is uniquely solvable in the class $C^{N}\left(t \geqslant 0, E_{1}\right)$ if and only if the conditions

$$
\begin{aligned}
(I-Q)[ & A u_{j}+f^{(j)}(0)+\Lambda(0) f^{(j-1)}(0)+\Lambda^{\prime}(0) f^{(j-2)}(0)+\ldots \\
& \left.+\Lambda^{(j-2)}(0) f^{\prime}(0)+\Lambda^{(j-1)}(0) f(0)\right]=0, \quad j=0,1, \ldots, N-1,
\end{aligned}
$$

are satisfied.

Example 1. We consider the following initial boundary value problem of visco-elasticity theory [10]

$$
(\lambda-\Delta) u_{t t}-(\mu-\Delta) u-\int_{0}^{t} g(t-\tau)(\gamma-\Delta) u(\tau, \bar{x}) d \tau=f(t, \bar{x})
$$




$$
\left.u\right|_{t=0}=u_{0}(\bar{x}),\left.\quad u_{t}\right|_{t=0}=u_{1}(\bar{x}), \quad \bar{x} \in \Omega ;\left.\quad u\right|_{\bar{x} \in \partial \Omega}=0, \quad t \geqslant 0,
$$

where $g(t), f(t, \bar{x})$ are given functions, $u=u(t, \bar{x})$ is a sought function, $\bar{x} \in \Omega \subset \mathbb{R}^{m}$ is a bounded domain with an infinitely differentiable boundary $\partial \Omega, \Delta$ is the Laplace operator, $u=u(t, \bar{x})$ is defined on a cylinder $\mathbb{R}_{+} \times \Omega, \lambda \in \sigma(\Delta)$.

For Dirichlet-Cauchy problem (8) - (9) with $\mu \neq \lambda, \lambda \in \sigma(\Delta)$, we define the Banach spaces and operators as follows:

$$
E_{1} \equiv\left\{v(\bar{x}) \in W_{p}^{k+2}(\Omega):\left.v\right|_{\partial \Omega}=0\right\}, \quad E_{2} \equiv W_{p}^{k}(\Omega), \quad B=\lambda-\Delta, \quad A=\mu-\Delta,
$$

where $W_{p}^{k}(\Omega)$ are Sobolev spaces. Under these notations, the kernel of the integral operator in equation (8) is represented as

$$
g(t)(\gamma-\Delta)=\alpha(t) A+\beta(t) B
$$

where

$$
\alpha(t)=\frac{\gamma-\lambda}{\mu-\lambda} g(t), \quad \beta(t)=\frac{\mu-\gamma}{\mu-\lambda} g(t),
$$

the operator $A$ is spectrally relatively bounded with respect to $B$ and $\infty$ is a removable singular point of the operator pencil $(\mu B-A)^{-1}$, see [4], [9] of the operator pencil. Then, according Theorem 2, the following statement holds true.

Theorem 3. Assume that for Dirichlet Cauchy problem (8)-(9) with $\mu \neq \lambda, \lambda \in \sigma(\Delta)$ the Banach space $E_{1}, E_{2}$, the operators $A, B$ are defined according (10). Then there exists a unique solution $u(t) \in C^{2}\left(t \geqslant 0, E_{1}\right)$ to problem (8)-(9) if and only if initial boundary conditions (9) and the function $f(t, \bar{x})$ satisfy the conditions

$$
\begin{aligned}
& \left\langle(\mu-\lambda) u_{0}(\bar{x})+f(0, \bar{x}), \varphi_{i}(\bar{x})\right\rangle=0, \\
& \left\langle(\mu-\lambda)^{2} u_{1}(\bar{x})+(\mu-\lambda) f_{t}^{\prime}(0, \bar{x})-(\gamma-\lambda) g(0) f(0, \bar{x}), \varphi_{i}(\bar{x})\right\rangle=0, \quad i=1, \ldots, n .
\end{aligned}
$$

Here $\varphi_{i}(\bar{x}), i=1, \ldots, n$, is an orthonormalized basis in the space of solutions to the homogenized problem $\lambda \varphi_{i}=\Delta \varphi_{i},\left.\varphi_{i}\right|_{\bar{x} \in \partial \Omega}=0$.

Remark 6. Solvability conditions of Dirichlet Cauchy problem (8)-(9) can be rewritten as follows

$$
\begin{aligned}
& \left\langle(\mu-\lambda) u_{0}(\bar{x})+f(0, \bar{x}), \varphi_{i}(\bar{x})\right\rangle=0 \\
& \left\langle(\mu-\lambda) u_{1}(\bar{x})+(\gamma-\lambda) g(0) u_{0}(\bar{x})+f_{t}^{\prime}(0, \bar{x}), \varphi_{i}(\bar{x})\right\rangle=0, \quad i=1, \ldots, n .
\end{aligned}
$$

\section{FUNDAMENTAL OPERATOR FUNCTIONS UNDER POLYNOMIAL BOUNDEDNESS}

Let $E_{1}, E_{2}$ be Banach spaces, $B, A_{1}, A_{0} \in L\left(E_{1}, E_{2}\right)$, the operator $B$ is non-invertible. Following work [3], we introduce a series of notions: a $B$-resolvent set of the pair of operators $\left(A_{1}, A_{0}\right)$ is a following open set in the complex plane:

$$
\rho^{B}\left(A_{1}, A_{0}\right) \equiv\left\{\mu \in C: R_{\mu}^{B}\left(A_{1}, A_{0}\right)=\left(\mu^{2} B-\mu A_{1}-A_{0}\right)^{-1} \in L\left(E_{2}, E_{1}\right)\right\} .
$$

A pair of operators $\left(A_{1}, A_{0}\right)$ is called polynomially relatively bounded with respect to the operator $B$ (or polynomially $B$-bounded) if there exists a number $a>0$ such that

$$
\{\mu \in \mathbb{C}:|\mu|>a\} \subset \rho^{B}\left(A_{1}, A_{0}\right) .
$$

We consider a circumference $\Gamma \equiv\{\mu \in \mathbb{C}:|\mu|=r>a\}$, then under the polynomial $B$ boundedness we shall assume the following condition:

A) for each circumference $\Gamma$ of an aforementioned form we have

$$
\oint_{\Gamma} R_{\mu}^{B}\left(A_{1}, A_{0}\right) d \mu \equiv 0
$$


In this case [3], the operators

$$
\tilde{P}=\frac{1}{2 \pi i} \oint_{\Gamma} \mu R_{\mu}^{B}\left(A_{1}, A_{0}\right) B d \mu, \quad \tilde{Q}=\frac{1}{2 \pi i} \oint_{\Gamma} \mu B R_{\mu}^{B}\left(A_{1}, A_{0}\right) d \mu
$$

are projectors in $E_{1}$ and $E_{2}$, respectively, and they give rise to expansions of these spaces into direct sums:

$$
E_{1} \equiv E_{1}^{0} \oplus E_{1}^{1}=\operatorname{Ker} \tilde{P} \oplus \operatorname{Im} \tilde{P}
$$

and

$$
E_{2} \equiv E_{2}^{0} \oplus E_{2}^{1}=\operatorname{Ker} \tilde{Q} \oplus \operatorname{Im} \tilde{Q}
$$

The action of the operators $B, A_{1}, A_{0}$ are split and the restrictions $A_{0}^{0}: E_{1}^{0} \rightarrow E_{2}^{0}$ and $B_{1}: E_{1}^{1} \rightarrow E_{2}^{1}$ are continuously invertible.

In what follows, we also assume a pseudo-commutation condition:

B) The operators $B$ and $A_{1}$ pseudo-commute with respect to $R_{\mu}^{B}\left(A_{1}, A_{0}\right)$, that is, for all $\mu \in \rho^{B}\left(A_{1}, A_{0}\right)$ the identity

$$
B R_{\mu}^{B}\left(A_{1}, A_{0}\right) A_{1}=A_{1} R_{\mu}^{B}\left(A_{1}, A_{0}\right) B
$$

holds true.

As it was shown in [3], if condition $\mathbf{B}$ ) is satisfied, then the pairs of operators $B$ and $A_{0}, A_{1}$ and $A_{0}$ also pseudo-commute with respect to $R_{\mu}^{B}\left(A_{1}, A_{0}\right)$.

We then assume that $\alpha_{0}(t), \alpha_{1}(t), \beta(t) \in C(t \geqslant 0), \Lambda_{0}(t)$ is the resolvent of the kernel $\left(-\alpha_{0}(t) \theta(t)\right), R_{1}(t)$ is the resolvent of the convolution kernel

$$
k_{1}(t) \theta(t)=f_{2 N}(t) * \beta(t) \theta(t),
$$

and

$$
g(t) \theta(t)=f_{N}(t) * \alpha_{1}(t) \theta(t)
$$

$N$ is a natural number.

We introduce two recurrent families of generalized operator functions:

$$
\begin{aligned}
& K_{0}^{1}(t)=I \delta(t), \quad K_{0}^{2}(t)=I \delta(t), \\
& K_{1}^{1}(t)=H_{0}(t)=\left(\delta(t)+\Lambda_{0}(t) \theta(t)\right) *\left(\delta(t)-k_{1}(t) \theta(t)\right)\left(A_{0}^{0}\right)^{-1} B_{0}, \\
& K_{1}^{2}(t)=-H_{1}(t)=-\left(\delta(t)+\Lambda_{0}(t) \theta(t)\right) *(\delta(t)+g(t) \theta(t))\left(A_{0}^{0}\right)^{-1} A_{1}^{0}, \\
& K_{q+1}^{1}(t)=K_{q}^{2}(t) * H_{0}(t), \quad K_{q+1}^{2}(t)=K_{q}^{1}(t)-K_{q}^{2}(t) * H_{1}(t)
\end{aligned}
$$

and

$$
\begin{aligned}
& L_{0}^{1}(t)=I \delta(t), \quad L_{0}^{2}(t)=I \delta(t), \\
& L_{1}^{1}(t)=S_{0}(t)=\left(\delta(t)+R_{1}(t) \theta(t)\right) *\left(\delta(t)+\alpha_{0}(t) \theta(t)\right) B_{1}^{-1} A_{0}^{1}, \\
& \left.L_{1}^{2}(t)=S_{1}(t)=\left(\delta(t)+R_{1}(t)\right) \theta(t)\right) *(\delta(t)+g(t) \theta(t)) B_{1}^{-1} A_{1}^{1}, \\
& L_{q+1}^{1}(t)=L_{q}^{2}(t) * S_{0}(t), \quad L_{q+1}^{2}(t)=L_{q}^{1}(t)+L_{q}^{2}(t) * S_{1}(t) .
\end{aligned}
$$

Theorem 4. If condition A) is satisfied, then the following convolution identities hold:

$$
\begin{aligned}
& \left(\delta(t)-k_{1}(t) \theta(t)\right) B * K_{q}^{i}(t)-(\delta(t)+g(t) \theta(t)) A_{1} * K_{q+1}^{i}(t) \\
& \quad-\left(\delta(t)+\alpha_{0}(t) \theta(t)\right) A_{0} * K_{q+2}^{i}(t) \equiv 0, \\
& \left(\delta(t)-k_{1}(t) \theta(t)\right) B * L_{q+2}^{i}(t)-(\delta(t)+g(t) \theta(t)) A_{1} * L_{q+1}^{i}(t) \\
& \quad-\left(\delta(t)+\alpha_{0}(t) \theta(t)\right) A_{0} * L_{q}^{i}(t) \equiv 0, \quad i=1,2, \quad q=0,1,2, \ldots, \\
& K_{q+k+2}^{2}(t)=K_{k+1}^{1}(t) * K_{q}^{2}(t)+K_{k+1}^{2}(t) * K_{q+1}^{2}(t), \\
& L_{q+k+2}^{2}(t)=L_{k+1}^{1}(t) * L_{q}^{2}(t)+L_{k+1}^{2}(t) * L_{q+1}^{2}(t), \quad k, q=0,1,2, \ldots
\end{aligned}
$$


Proof. We are going to prove these identities by induction in $q$. As $q=0$ and $q=1$, the identities can be confirmed by straightforward calculations. Let $g \geqslant 2$, then

$$
\begin{aligned}
\left(\delta(t)-k_{1}(t) \theta(t)\right) B * & K_{q}^{2}(t)-(\delta(t)+g(t) \theta(t)) A_{1} * K_{q+1}^{2}(t) \\
& -\left(\delta(t)+\alpha_{0}(t) \theta(t)\right) A_{0} * K_{q+2}^{2}(t) \\
= & \left(\delta(t)-k_{1}(t) \theta(t)\right) B *\left(K_{q-1}^{1}(t)-K_{q-1}^{2}(t) * H_{1}(t)\right) \\
& -(\delta(t)+g(t) \theta(t)) A_{1} *\left(K_{q}^{1}(t)-K_{q}^{2}(t) * H_{1}(t)\right) \\
& -\left(\delta(t)+\alpha_{0}(t) \theta(t)\right) A_{0} *\left(K_{q+1}^{1}(t)-K_{q+1}^{2}(t) * H_{1}(t)\right) \\
= & {\left[\left(\delta(t)-k_{1}(t) \theta(t)\right) B * K_{q-2}^{2}(t)-(\delta(t)+g(t) \theta(t)) A_{1} * K_{q-1}^{2}(t)\right.} \\
& \left.-\left(\delta(t)+\alpha_{0}(t) \theta(t)\right) A_{0} * K_{q}^{2}(t)\right] * H_{0}(t) \\
& -\left[\left(\delta(t)-k_{1}(t) \theta(t)\right) B * K_{q-1}^{2}(t)-(\delta(t)+g(t) \theta(t)) A_{1} * K_{q}^{2}(t)\right. \\
& \left.-\left(\delta(t)+\alpha_{0}(t) \theta(t)\right) A_{0} * K_{q+1}^{2}(t)\right] * H_{1}(t) \equiv 0,
\end{aligned}
$$

and

$$
\begin{aligned}
K_{q+k+2}^{2}(t)= & K_{q+k+1}^{1}(t)-K_{q+k+1}^{2}(t) * H_{1}(t) \\
= & K_{q+k}^{2}(t) * H_{0}(t)-K_{q+k+1}^{2}(t) * H_{1}(t) \\
= & \left(K_{k+1}^{1}(t) * K_{q-2}^{2}(t)+K_{k+1}^{2}(t) * K_{q-1}^{2}(t)\right) * H_{0}(t) \\
& -\left(K_{k+1}^{1}(t) * K_{q-1}^{2}(t)+K_{k+1}^{2}(t) * K_{q}^{2}(t)\right) * H_{1}(t) \\
= & K_{k+1}^{1}(t) *\left[K_{q-2}^{2}(t) * H_{0}(t)-K_{q-1}^{2}(t) * H_{1}(t)\right] \\
& +K_{k+1}^{2}(t) *\left[K_{q-1}^{2}(t) * H_{0}(t)-K_{q}^{2}(t) * H_{1}(t)\right] \\
= & K_{k+1}^{1}(t) *\left[K_{q-1}^{1}(t)-K_{q-1}^{2}(t) * H_{1}(t)\right]+K_{k+1}^{2}(t) *\left[K_{q}^{1}(t)-K_{q}^{2}(t) * H_{1}(t)\right] \\
= & K_{k+1}^{1}(t) * K_{q}^{2}(t)+K_{k+1}^{2}(t) * K_{q+1}^{2}(t) .
\end{aligned}
$$

Other relations can be proved in the same way. The proof is complete.

Theorem 5. If a pair of operators $\left(A_{1}, A_{0}\right)$ is polynomially B-bounded, conditions $\left.\mathbf{A}\right)$ and B) are satisfied, then the integro-differential operator

$$
\begin{aligned}
B \delta^{(2 N)}(t) & -A_{1} \delta^{(N)}(t)-A_{0} \delta(t)-\left(\beta(t) B+\alpha_{1}(t) A_{1}+\alpha_{0}(t) A_{0}\right) \theta(t) \\
= & B\left(\delta^{(2 N)}(t)-\beta(t) \theta(t)\right)-A_{1}\left(\delta^{(N)}(t)+\alpha_{1}(t) \theta(t)\right)-A_{0}\left(\delta(t)+\alpha_{0}(t) \theta(t)\right)
\end{aligned}
$$

has a fundamental operator function

$$
\tilde{\mathcal{E}}_{N}(t)=\tilde{\mathcal{U}}_{N}(t) \tilde{Q}-\tilde{\mathcal{V}}_{N}(t)(I-\tilde{Q}),
$$

on the class $K_{+}^{\prime}\left(E_{2}\right)$. Here

$$
\begin{aligned}
& \tilde{\mathcal{U}}_{N}(t)=\left(\delta(t)+R_{1}(t) \theta(t)\right) * \sum_{q=0}^{\infty} f_{N \cdot(q+2)}(t) * L_{q}^{2}(t) B_{1}^{-1} \\
& \tilde{\mathcal{V}}_{N}(t)=\left(\delta(t)+\Lambda_{0}(t) \theta(t)\right) * \sum_{q=0}^{\infty} \delta^{(q \cdot N)}(t) * K_{q}^{2}(t)\left(A_{0}^{0}\right)^{-1} .
\end{aligned}
$$

Proof. We follow the lines of the proof of Theorem 1. First,

$$
B\left(\delta^{(2 N)}(t)-\beta(t) \theta(t)\right) * \tilde{\mathcal{U}}_{N}(t) \tilde{Q}
$$




$$
\begin{aligned}
= & B\left(\delta^{(2 N)}(t)-\beta(t) \theta(t)\right) *\left(\delta(t)+R_{1}(t) \theta(t)\right) * f_{2 N}(t) * B_{1}^{-1} \tilde{Q} \delta(t) \\
& +\left(\delta(t)+R_{1}(t) \theta(t)\right) * \sum_{q=1}^{\infty} f_{N \cdot(q+2)}(t) *\left(\delta^{(2 N)}(t)-\beta(t) \theta(t)\right) B * L_{q}^{2}(t) B_{1}^{-1} \tilde{Q} \\
= & B\left(\delta(t)-k_{1}(t) \theta(t)\right) *\left(\delta(t)+R_{1}(t) \theta(t)\right) * B_{1}^{-1} \tilde{Q} \delta(t) \\
& +\left(\delta(t)+R_{1}(t) \theta(t)\right) * \sum_{q=1}^{\infty} f_{N \cdot q}(t) *\left(\delta(t)-k_{1}(t) \theta(t)\right) B * L_{q}^{2}(t) B_{1}^{-1} \tilde{Q} \\
= & \tilde{Q} \delta(t)+\left(\delta(t)+R_{1}(t) \theta(t)\right) *\left[f_{N}(t) *(\delta(t)+g(t) \theta(t)) A_{1}^{1} B_{1}^{-1} \tilde{Q}\right. \\
& \left.+\sum_{q=2}^{\infty} f_{N \cdot q}(t) *\left(\delta(t)-k_{1}(t) \theta(t)\right) B * L_{q}^{2}(t) B_{1}^{-1} \tilde{Q}\right] .
\end{aligned}
$$

Second,

$$
\begin{aligned}
A_{1}\left(\delta^{(N)}(t)+\alpha_{1}(t) \theta(t)\right) * \tilde{\mathcal{U}}_{N}(t) \tilde{Q} \\
=\left(\delta(t)+R_{1}(t) \theta(t)\right) * \sum_{q=0}^{\infty} f_{N \cdot(q+1)}(t) *(\delta(t)+g(t) \theta(t)) A_{1} * L_{q}^{2}(t) B_{1}^{-1} \tilde{Q} \\
=\left(\delta(t)+R_{1}(t) \theta(t)\right) *\left[f_{N}(t) *(\delta(t)+g(t) \theta(t)) A_{1}^{1} B_{1}^{-1} \tilde{Q}\right. \\
\left.+\sum_{q=1}^{\infty} f_{N \cdot(q+1)}(t) *(\delta(t)+g(t) \theta(t)) A_{1} * L_{q}^{2}(t) B_{1}^{-1} \tilde{Q}\right]
\end{aligned}
$$

Third,

$$
\begin{aligned}
& A_{0}\left(\delta(t)+\alpha_{0}(t) \theta(t)\right) * \tilde{\mathcal{U}}_{N}(t) \tilde{Q} \\
& \quad=\left(\delta(t)+R_{1}(t) \theta(t)\right) * \sum_{q=0}^{\infty} f_{N \cdot(q+2)}(t) *\left(\delta(t)+\alpha_{0}(t) \theta(t)\right) A_{0} * L_{q}^{2}(t) B_{1}^{-1} \tilde{Q} .
\end{aligned}
$$

By identity (14) this implies

$$
\begin{aligned}
& {\left[B\left(\delta^{(2 N)}(t)-\beta(t) \theta(t)\right)-A_{1}\left(\delta^{(N)}(t)+\alpha_{1}(t) \theta(t)\right)\right.} \\
& \left.\quad-A_{0}\left(\delta(t)+\alpha_{0}(t) \theta(t)\right)\right] * \tilde{\mathcal{U}}_{N}(t) \tilde{Q} \\
& =\tilde{Q} \delta(t)+\left(\delta(t)+R_{1}(t) \theta(t)\right) * \sum_{q=0}^{\infty} f_{N \cdot(q+2)}(t) *\left[\left(\delta(t)-k_{1}(t) \theta(t)\right) B * L_{q+2}^{2}(t)\right. \\
& \left.\quad-(\delta(t)+g(t) \theta(t)) A_{1} * L_{q+1}^{2}(t)-\left(\delta(t)+\alpha_{0}(t) \theta(t)\right) A_{0} * L_{q}^{2}(t)\right] B_{1}^{-1} \tilde{Q} \\
& =\tilde{Q} \delta(t) .
\end{aligned}
$$

Then, first,

$$
B\left(\delta^{(2 N)}(t)-\beta(t) \theta(t)\right) * \tilde{\mathcal{V}}_{N}(t)(I-\tilde{Q})
$$




$$
\begin{aligned}
= & \left(\delta(t)+\Lambda_{0}(t) \theta(t)\right) * \sum_{q=0}^{\infty} \delta^{(q \cdot N)}(t) *\left(\delta^{(2 N)}(t)-\beta(t) \theta(t)\right) B * K_{q}^{2}(t)\left(A_{0}^{0}\right)^{-1}(I-\tilde{Q}) \\
= & \left(\delta(t)+\Lambda_{0}(t) \theta(t)\right) * \sum_{q=0}^{\infty} \delta^{((q+2) \cdot N)}(t) * f_{2 N}(t) *\left(\delta^{(2 N)}(t)-\beta(t) \theta(t)\right) B \\
& * K_{q}^{2}(t)\left(A_{0}^{0}\right)^{-1}(I-\tilde{Q}) \\
= & \left(\delta(t)+\Lambda_{0}(t) \theta(t)\right) * \sum_{q=0}^{\infty} \delta^{((q+2) \cdot N)}(t) *\left(\delta(t)-k_{1}(t) \theta(t)\right) B * K_{q}^{2}(t)\left(A_{0}^{0}\right)^{-1}(I-\tilde{Q}) .
\end{aligned}
$$

Second,

$$
\begin{aligned}
& A_{1}\left(\delta^{(N)}(t)+\alpha_{1}(t) \theta(t)\right) * \tilde{\mathcal{V}}_{N}(t)(I-\tilde{Q}) \\
& =\left(\delta(t)+\Lambda_{0}(t) \theta(t)\right) * \sum_{q=0}^{\infty} \delta^{(q \cdot N)}(t) *\left(\delta^{(N)}(t)+\alpha_{1}(t) \theta(t)\right) A_{1} * K_{q}^{2}(t)\left(A_{0}^{0}\right)^{-1}(I-\tilde{Q}) \\
& =\left(\delta(t)+\Lambda_{0}(t) \theta(t)\right) * \sum_{q=0}^{\infty} \delta^{((q+1) \cdot N)}(t) * f_{N}(t) *\left(\delta^{(N)}(t)+\alpha_{1}(t) \theta(t)\right) A_{1} \\
& \quad * K_{q}^{2}(t)\left(A_{0}^{0}\right)^{-1}(I-\tilde{Q}) \\
& =\left(\delta(t)+\Lambda_{0}(t) \theta(t)\right) *\left[\sum_{q=1}^{\infty} \delta^{((q+1) \cdot N)}(t) *(\delta(t)+g(t) \theta(t)) A_{1} * K_{q}^{2}(t)\right. \\
& \left.\quad+\delta^{(N)}(t) *(\delta(t)+g(t) \theta(t)) A_{1}^{0}\right]\left(A_{0}^{0}\right)^{-1}(I-\tilde{Q}) .
\end{aligned}
$$

And third,

$$
\begin{aligned}
A_{0}( & \left.\delta(t)+\alpha_{0}(t) \theta(t)\right) * \tilde{\mathcal{V}}_{N}(t)(I-\tilde{Q}) \\
= & (I-\tilde{Q}) \delta(t)+\left(\delta(t)+\Lambda_{0}(t) \theta(t)\right) * \sum_{q=1}^{\infty} \delta^{(q \cdot N)}(t) *\left(\delta(t)+\alpha_{0}(t) \theta(t)\right) A_{0} \\
& * K_{q}^{2}(t)\left(A_{0}^{0}\right)^{-1}(I-\tilde{Q}) \\
= & (I-\tilde{Q}) \delta(t)+\left(\delta(t)+\Lambda_{0}(t) \theta(t)\right) *\left[\sum_{q=2}^{\infty} \delta^{(q \cdot N)}(t) *\left(\delta(t)+\alpha_{0}(t) \theta(t)\right) A_{0} * K_{q}^{2}(t)\right. \\
& \left.-\delta^{(N)}(t) *(\delta(t)+g(t) \theta(t)) A_{1}^{0}\right]\left(A_{0}^{0}\right)^{-1}(I-\tilde{Q}) .
\end{aligned}
$$

By identity (13) this yields:

$$
\begin{aligned}
& {\left[B\left(\delta^{(2 N)}(t)-\beta(t) \theta(t)\right)-A_{1}\left(\delta^{(N)}(t)+\alpha_{1}(t) \theta(t)\right)\right.} \\
& \left.-A_{0}\left(\delta(t)+\alpha_{0}(t) \theta(t)\right)\right] * \tilde{\mathcal{V}}_{N}(t)(I-\tilde{Q}) \\
= & -(I-\tilde{Q}) \delta(t)+\left(\delta(t)+\Lambda_{0}(t) \theta(t)\right) * \sum_{q=0}^{\infty} \delta^{(N(q+2))}(t) *\left[\left(\delta(t)-k_{1}(t) \theta(t)\right) B * K_{q}^{2}(t)\right. \\
& \left.-(\delta(t)+g(t) \theta(t)) A_{1} * K_{q+1}^{2}(t)-\left(\delta(t)+\alpha_{0}(t) \theta(t)\right) A_{0} * K_{q+2}^{2}(t)\right]\left(A_{0}^{0}\right)^{-1}(I-\tilde{Q})
\end{aligned}
$$




$$
=-(I-\tilde{Q}) \delta(t)
$$

Hence,

$$
\begin{gathered}
{\left[B\left(\delta^{(2 N)}(t)-\beta(t) \theta(t)\right)-A_{1}\left(\delta^{(N)}(t)+\alpha_{1}(t) \theta(t)\right)-A_{0}\left(\delta(t)+\alpha_{0}(t) \theta(t)\right)\right] * \tilde{\mathcal{E}}_{N}(t)} \\
=\tilde{Q} \delta(t)+(I-\tilde{Q}) \delta(t)=I \delta(t) .
\end{gathered}
$$

By similar arguing we find that, first,

$$
\begin{aligned}
\tilde{\mathcal{U}}_{N}(t) \tilde{Q} * B\left(\delta^{(2 N)}(t)-\beta(t) \theta(t)\right) \\
=\left(\delta(t)+R_{1}(t) \theta(t)\right) * \sum_{q=0}^{\infty} f_{N \cdot q}(t) *\left(\delta(t)-k_{1}(t) \theta(t)\right) * L_{q}^{2}(t) \tilde{P} \\
=\sum_{q=0}^{\infty} f_{N \cdot q}(t) * L_{q}^{2}(t) \tilde{P}=\tilde{P} \delta(t)+\left[f_{N}(t) * L_{1}^{2}(t)+\sum_{q=2}^{\infty} f_{N \cdot q}(t) * L_{q}^{2}(t)\right] \tilde{P} .
\end{aligned}
$$

Second,

$$
\begin{aligned}
\tilde{\mathcal{U}}_{N}(t) & \tilde{Q} * A_{1}\left(\delta^{(N)}(t)+\alpha_{1}(t) \theta(t)\right) \\
& =\left(\delta(t)+R_{1}(t) \theta(t)\right) * \sum_{q=0}^{\infty} f_{N \cdot(q+1)}(t) *(\delta(t)+g(t) \theta(t)) * L_{q}^{2}(t) B_{1}^{-1} A_{1}^{1} \tilde{P} \\
& =\sum_{q=0}^{\infty} f_{N \cdot(q+1)}(t) * L_{q}^{2}(t) * S_{1}(t) \tilde{P} \\
& =\left[f_{N}(t) * L_{1}^{2}(t)+\sum_{q=1}^{\infty} f_{N \cdot(q+1)}(t) * L_{q}^{2}(t) * S_{1}(t)\right] \tilde{P} .
\end{aligned}
$$

And third,

$$
\tilde{\mathcal{U}}_{N}(t) \tilde{Q} * A_{0}\left(\delta(t)+\alpha_{0}(t) \theta(t)\right)=\sum_{q=0}^{\infty} f_{N \cdot(q+2)}(t) * L_{q}^{2}(t) * S_{0}(t) \tilde{P} .
$$

By recurrent relations 12 this implies:

$$
\begin{aligned}
\tilde{\mathcal{U}}_{N}(t) \tilde{Q} * & {\left[B\left(\delta^{(2 N)}(t)-\beta(t) \theta(t)\right)-A_{1}\left(\delta^{(N)}(t)+\alpha_{1}(t) \theta(t)\right)\right.} \\
& \left.-A_{0}\left(\delta(t)+\alpha_{0}(t) \theta(t)\right)\right] \\
= & \tilde{P} \delta(t)+\sum_{q=0}^{\infty} f_{N \cdot(q+2)}(t) *\left(L_{q+2}^{2}(t)-L_{q+1}^{2}(t) * S_{1}(t)-L_{q}^{2}(t) * S_{0}(t)\right) \tilde{P} \\
= & \tilde{P} \delta(t)+\sum_{q=0}^{\infty} f_{N \cdot(q+2)}(t) *\left(L_{q+2}^{2}(t)-L_{q+1}^{2}(t) * S_{1}(t)-L_{q+1}^{1}(t)\right) \tilde{P} \\
= & \tilde{P} \delta(t) .
\end{aligned}
$$


In order to complete the proof, it remains to obtain the following three relations. The first reads as

$$
\begin{aligned}
\tilde{\mathcal{V}}_{N}(t) & (I-\tilde{Q}) * B\left(\delta^{(2 N)}(t)-\beta(t) \theta(t)\right) \\
= & \left(\delta(t)+\Lambda_{0}(t) \theta(t)\right) * \sum_{q=0}^{\infty} \delta^{(N \cdot q)}(t) *\left(\delta^{(2 N)}(t)-\beta(t) \theta(t)\right) * K_{q}^{2}(t)\left(A_{0}^{0}\right)^{-1} B_{0}(I-\tilde{P}) \\
= & \left(\delta(t)+\Lambda_{0}(t) \theta(t)\right) * \sum_{q=0}^{\infty} \delta^{(N \cdot(q+2))}(t) *\left(\delta(t)-k_{1}(t) \theta(t)\right) * K_{q}^{2}(t)\left(A_{0}^{0}\right)^{-1} B_{0}(I-\tilde{P}) \\
= & {\left[\sum_{q=0}^{\infty} \delta^{(N \cdot(q+2))}(t) * K_{q}^{2}(t) * H_{0}(t)\right](I-\tilde{P}) . }
\end{aligned}
$$

The second relation is

$$
\begin{aligned}
\tilde{\mathcal{V}}_{N}(t) & (I-\tilde{Q}) * A_{1}\left(\delta^{(N)}(t)+\alpha_{1}(t) \theta(t)\right) \\
= & \left(\delta(t)+\Lambda_{0}(t) \theta(t)\right) * \sum_{q=0}^{\infty} \delta^{(N \cdot q)}(t) *\left(\delta^{(N)}(t)+\alpha_{1}(t) \theta(t)\right) * K_{q}^{2}(t)\left(A_{0}^{0}\right)^{-1} A_{1}^{0}(I-\tilde{P}) \\
= & \left(\delta(t)+\Lambda_{0}(t) \theta(t)\right) * \sum_{q=0}^{\infty} \delta^{(N \cdot(q+1))}(t) *(\delta(t)+g(t) \theta(t)) * K_{q}^{2}(t)\left(A_{0}^{0}\right)^{-1} A_{1}^{0}(I-\tilde{P}) \\
= & {\left[\sum_{q=0}^{\infty} \delta^{(N \cdot(q+1))}(t) * K_{q}^{2}(t) * H_{1}(t)\right](I-\tilde{P}) } \\
= & {\left[\delta^{(N)}(t) * H_{1}(t)+\sum_{q=1}^{\infty} \delta^{(N \cdot(q+1))}(t) * K_{q}^{2}(t) * H_{1}(t)\right](I-\tilde{P}) . }
\end{aligned}
$$

And the third identity is as follows:

$$
\begin{aligned}
\tilde{\mathcal{V}}_{N}(t)(I-\tilde{Q}) * A_{0}\left(\delta^{(N)}(t)+\alpha_{0}(t) \theta(t)\right) \\
=\left(\delta(t)+\Lambda_{0}(t) \theta(t)\right) * \sum_{q=0}^{\infty} \delta^{(N \cdot q)}(t) *\left(\delta(t)+\alpha_{0}(t) \theta(t)\right) * K_{q}^{2}(t)(I-\tilde{P}) \\
=\sum_{q=0}^{\infty} \delta^{(N \cdot q)}(t) * K_{q}^{2}(t)(I-\tilde{P}) \\
=(I-\tilde{P}) \delta(t)+\left[\delta^{(N)}(t) * K_{1}^{2}(t)+\sum_{q=2}^{\infty} \delta^{(N \cdot q)}(t) * K_{q}^{2}(t)\right](I-\tilde{P}) \\
=(I-\tilde{P}) \delta(t)+\left[-\delta^{(N)}(t) * H_{1}(t)+\sum_{q=2}^{\infty} \delta^{(N \cdot q)}(t) * K_{q}^{2}(t)\right](I-\tilde{P}) .
\end{aligned}
$$

According recurrent relations (11) we have:

$$
\begin{aligned}
\tilde{\mathcal{V}}_{N}(t) & (I-\tilde{Q}) *\left[B\left(\delta^{(2 N)}(t)-\beta(t) \theta(t)\right)-A_{1}\left(\delta^{(N)}(t)+\alpha_{1}(t) \theta(t)\right)\right. \\
& \left.-A_{0}\left(\delta(t)+\alpha_{0}(t) \theta(t)\right)\right]
\end{aligned}
$$




$$
\begin{aligned}
= & \sum_{q=0}^{\infty} \delta^{(N \cdot(q+2))}(t) *\left[K_{q}^{2}(t) * H_{0}(t)-K_{q+1}^{2}(t) * H_{1}(t)-K_{q+2}^{2}(t)\right](I-\tilde{P}) \\
& -(I-\tilde{P}) \delta(t) \\
= & \sum_{q=0}^{\infty} \delta^{(N \cdot(q+2))}(t) *\left[K_{q+1}^{1}(t)-K_{q+1}^{2}(t) * H_{1}(t)-K_{q+2}^{2}(t)\right](I-\tilde{P})-(I-\tilde{P}) \delta(t) \\
= & -(I-\tilde{P}) \delta(t) .
\end{aligned}
$$

We finally obtain:

$$
\begin{aligned}
\tilde{\mathcal{E}}_{N}(t) & *\left[B\left(\delta^{(2 N)}(t)-\beta(t) \theta(t)\right)-A_{1}\left(\delta^{(N)}(t)+\alpha_{1}(t) \theta(t)\right)\right. \\
& \left.-A_{0}\left(\delta(t)+\alpha_{0}(t) \theta(t)\right)\right]=\tilde{P} \delta(t)+(I-\tilde{P}) \delta(t)=I \delta(t) .
\end{aligned}
$$

\section{Remark 7. If}

$$
\alpha_{0}(t)=\alpha_{1}(t)=\beta(t) \equiv 0
$$

in equation (3), our Theorem 5 coincides with Theorem 5 in work [5], and if

$$
\alpha_{1}(t)=\beta(t) \equiv 0,
$$

then it coincides with Theorem 5 in work [6]. The conclusions formulated in Remark 5 on Cauchy problem (1)-(2) are also true for Cauchy problem (3)-(4).

If $\infty$ is a removable singular point of the pair of operators $\left(A_{1}, A_{0}\right)$ polynomially relatively bounded with respect to $B$, see [3], that is, $K_{1}^{1} \equiv 0, K_{1}^{2} \equiv 0$ or $\left(A_{0}^{0}\right)^{-1} B_{0} \equiv 0,\left(A_{0}^{0}\right)^{-1} A_{1}^{0} \equiv 0$, then the fundamental operator function in Theorem 5 reads as

$$
\tilde{\tilde{\mathcal{E}}}_{N}(t)=\tilde{\mathcal{U}}_{N}(t) \tilde{Q}-\left(\delta(t)+\Lambda_{0}(t) \theta(t)\right)\left(A_{0}^{0}\right)^{-1}(I-\tilde{Q}) .
$$

Then the unique solution to Cauchy problem (3)-(4) is a regular generalized function

$$
\tilde{u}(t)=\tilde{\mathcal{E}}_{N}(t) *\left(f(t) \theta(t)+B u_{2 N-1} \delta(t)+B u_{2 N-2} \delta^{\prime}(t)+\cdots+B u_{1} \delta^{(2 N-2)}(t)+B u_{0} \delta^{(2 N-1)}(t)\right),
$$

which turns equation (3) into identity and belongs to the class $C^{2 N}\left(t \geqslant 0, E_{1}\right)$. The conditions, under which this function satisfies initial condition (4), are exactly the solvability conditions for Cauchy problem (3)-(4) in the class $C^{2 N}\left(t \geqslant 0, E_{1}\right)$. By straightforward calculations we find:

where

$$
\left.\tilde{u}^{(j)}\right|_{t=0}=u_{j}-\tilde{\omega}_{j}, \quad j=0,1,2, \ldots, 2 N-1,
$$

$$
\begin{aligned}
\tilde{\omega}_{j}= & \left(A_{0}^{0}\right)^{-1}(I-\tilde{Q})\left[A_{0} u_{j}+f^{(j)}(0)+\Lambda_{0}(0) f^{(j-1)}(0)+\Lambda_{0}^{\prime}(0) f^{(j-2)}(0)+\ldots\right. \\
& \left.+\Lambda_{0}^{(j-2)}(0) f^{\prime}(0)+\Lambda_{0}^{(j-1)}(0) f(0)\right] \\
= & \left(A_{0}^{0}\right)^{-1}(I-\tilde{Q}) \tilde{v}_{j} .
\end{aligned}
$$

Since $\left(A_{0}^{0}\right)^{-1} \in \mathcal{L}\left(E_{2}, E_{1}\right)$, then $\tilde{\omega}_{j}=0$ as $(I-\tilde{Q}) \tilde{v}_{j}=0$.

Theorem 6. If under the assumptions of Theorem $5 \infty$ is a removable singular point, then Cauchy problem (3)-(4) is uniquely solvable in the class $C^{2 N}\left(t \geqslant 0, E_{1}\right)$ if and only if the conditions hold:

$$
(I-\tilde{Q})\left[A_{0} u_{j}+f^{(j)}(0)+\Lambda_{0}(0) f^{(j-1)}(0)+\Lambda_{0}^{\prime}(0) f^{(j-2)}(0)+\ldots\right.
$$




$$
\left.+\Lambda_{0}^{(j-2)}(0) f^{\prime}(0)+\Lambda_{0}^{(j-1)}(0) f(0)\right]=0, \quad j=0,1,2, \ldots, 2 N-1 .
$$

Example 2. For an integro-differential analogue of equation of visco-elasticity theory [10] we consider the Dirichlet Cauchy problem:

$$
\begin{aligned}
& (\lambda-\Delta) u_{t t}-\beta(\mu-\Delta) u_{t}-\Delta^{2} u+\int_{0}^{t} g(t-\tau)\left(\gamma-\Delta^{2}\right) u(\tau, \bar{x}) d \tau=f(t, \bar{x}), \\
& \left.u\right|_{t=0}=u_{0}(\bar{x}),\left.\quad u_{t}\right|_{t=0}=u_{1}(\bar{x}), \quad \bar{x} \in \Omega ;\left.\quad u\right|_{\bar{x} \in \partial \Omega}=0, \quad t \geqslant 0,
\end{aligned}
$$

where $g(t), f(t, \bar{x})$ are given functions, $u=u(t, \bar{x})$ is a sought function, $\bar{x} \in \Omega \subset \mathbb{R}^{m}$ is a bounded domain with an infinitely smooth boundary $\partial \Omega, \Delta$ is the Laplace operator, $u=u(t, \bar{x})$ is defined on the cylinder $\mathbb{R}_{+} \times \Omega, \lambda \in \sigma(\Delta)$.

For Dirichlet Cauchy problem (15)-(16), where $\lambda \in \sigma(\Delta), \lambda \neq \mu$, we define Banach spaces $E_{1}$ and $E_{2}$ and operators as follows:

$$
\begin{array}{ll}
E_{1} \equiv\left\{v(\bar{x}) \in W_{p}^{k+4}(\Omega):\left.v\right|_{\partial \Omega}=0\right\}, & E_{2} \equiv W_{p}^{k}(\Omega), \\
B=\lambda-\Delta, \quad A_{1}=\beta(\mu-\Delta), & A_{0}=\Delta^{2},
\end{array}
$$

Then the kernel of the integral operator in equation $(15)$ is expanded into the sum

$$
-g(t)\left(\gamma-\Delta^{2}\right)=\beta(t)(\lambda-\Delta)+\alpha_{1}(t) \beta(\mu-\Delta)+\alpha_{0}(t) \Delta^{2},
$$

where

$$
\beta(t)=-\frac{\gamma}{\lambda-\mu} g(t), \quad \alpha_{1}(t)=\frac{\gamma}{\beta(\lambda-\mu)} g(t), \quad \alpha_{0}(t)=g(t) .
$$

Arguing as in [3, Lm. 4.2.1], we confirm that the chosen pair of the operators $\left(A_{1}, A_{0}\right)$ is polynomially relatively bounded with respect to $B$ and $\infty$ is a removable singular point. According Theorem 6 we obtain.

Theorem 7. Let for Dirichlet Cauchy problem (15)-(16) with $\lambda \in \sigma(\Delta), \lambda \neq \mu$ the Banach spaces $E_{1}$ and $E_{2}$ and the operators $B, A_{1}, A_{0}$ be defined as in (17). Then there exists the unique solution $u(t) \in C^{2 N}\left(t \geqslant 0, E_{1}\right)$ to problem (15)-16) if and only if initial boundary conditions (16) and the function $f(t, \bar{x})$ satisfy the relations

$$
\begin{aligned}
& \left\langle\lambda^{2} u_{0}(\bar{x})+f(0, \bar{x}), \varphi_{i}(\bar{x})\right\rangle=0, \\
& \left\langle\lambda^{2} u_{1}(\bar{x})+f_{t}^{\prime}(0, \bar{x})-g(0) f(0, \bar{x}), \varphi_{i}(\bar{x})\right\rangle=0, \quad i=1, \ldots, n .
\end{aligned}
$$

Here $\varphi_{i}(\bar{x}), i=1, \ldots, n$, is an orthonormalized basis in the space of solutions to a homogenized problem $\lambda \varphi_{i}=\Delta \varphi_{i},\left.\varphi_{i}\right|_{\bar{x} \in \partial \Omega}=0$.

Remark 8. As in Theorem 3, see Remark 6, the solvability condition of Dirichlet Cauchy problem (15)-(16) can be equivalently rewritten as

$$
\begin{aligned}
& \left\langle\lambda^{2} u_{0}(\bar{x})+f(0, \bar{x}), \varphi_{i}(\bar{x})\right\rangle=0, \\
& \left\langle\lambda^{2} u_{1}(\bar{x})+\lambda^{2} g(0) u_{0}(\bar{x})+f_{t}^{\prime}(0, \bar{x}), \varphi_{i}(\bar{x})\right\rangle=0, \quad i=1, \ldots, n .
\end{aligned}
$$

\section{BIBLIOGRAPHY}

1. V.S. Vladimirov. Generalized functions in mathematical physics. Nauka, Moscow (1979). [Mir Publishers, Moscow (1979).]

2. V.S. Vladimirov. Equations of mathematical physics. Nauka, Moscow (1982). [Pure Appl. Math. 3. Marcel Dekker, New York (1971).]

3. A.A. Zamyshlyaeva. Higher order linear equations of Sobolev type. Izd. centr YuURGU, Chelyabinsk (2012). (in Russian). 
4. G.A. Sviridyuk. On the general theory of operator semigroups // Uspekhi Matem. Nauk. 49:4, 47-74 (1994). [Russian Math. Surveys. 49:4, 45-74 (1994).]

5. M.V. Falaleev, E.Yu. Grazhdantseva. Fundamental operator functions of singular differential operators under spectral boundedness conditions // Diff. Uravn. 42:6, 769-774 (2006). [Differ. Equ. 42:6, 819-825 (2006).]

6. M.V. Falaleev, S.S. Orlov. Degenerated integro-differential equations of special kind in Banach spaces and its applications // Vestnik YuURGU. Ser. Matem. Model. Progr. 7:4, 100-110 (2011). (in Russian).

7. M.V. Falaleev. Linear models in theory of viscoelasticity of Sobolev type // Vestnik YuURGU. Ser. Matem. Model. Progr. 6:4, 101-107 (2013). (in Russian).

8. N. Sidorov, B. Loginov, A. Sinitsyn and M. Falaleev. Lyapunov-Schmidt methods in nonlinaear analysis and applications. Kluwer Acad. Publ., Dordrecht (2002).

9. G.A. Sviridyuk, V.E. Fedorov. Linear Sobolev type equations and degenerate semigroups of operators. VSP, Utrecht (2003).

10. M.M. Cavalcanti, V.N. Domingos Cavalcanti, J. Ferreira. Existence and uniform decay for a nonlinear viscoelastic equation with strong damping // Math. Meth. Appl. Sci. 24:14, 1043-1053 (2001).

Mikhail Valentinovich Falaleev,

Institute of Mathematics and Information Technologies,

Irkutsk State University,

Karl Marx str. 1,

664003, Irkutsk, Russia

E-mail: mvfalaleev@gmail.com 